\title{
Impact of Weak Organic Acids on Precipitation of Poly(acrylic acid) Stabilized Silver Nanoparticles; an Electrochemical Approach
}

\author{
Irena Ivanišević, Vanja Rukavina, Petar Kassal, Stjepan Milardović*
}

Department of General and Inorganic Chemistry, Faculty of Chemical Engineering and Technology, University of Zagreb, Zagreb, Croatia
* Corresponding author's e-mail address: stjepan.milardovic@fkit.hr

RECEIVED: October 24, 2018 * REVISED: February 15, 2019 * ACCEPTED: February 18, 2019

PROCEEDING OF THE $5^{\text {TH }}$ DAY OF Electrochemistry AND $8^{\text {TH }}$ ISE SSRSE, 25 MAY 2018, ZAGREB, CROATIA

\begin{abstract}
Owing to recent developments in the field of silver nanoparticle (AgNP) based conductive inks for inkjet printing, there is a growing demand for implementation of novel synthetic routes. This study presents a synthesis of silver colloid with an average particle diameter of less than $3 \mathrm{~nm}$. AgNPs were obtained by a chemical reduction method, using hydrazine as a reducing agent, and poly(acrylic acid), (PAA), as a grainstabilizing agent. Synthesis was provided using highly concentrated aqueous solutions. To obtain PAA-coated silver nanopowder, weak organic acids were introduced as precipitation agents. The main goal of this work was to study the impact of various organic precipitants on nanosurface-adsorbed polyacid for enhanced ink stability, by implementing electrochemical techniques. Cyclic voltammetry and electrochemical impedance spectroscopy were the main methods in describing all steps of the procedure - from preliminary experiments to whole system characterization. For a comprehensive study of the nanoink suspension, dynamic light scattering (DLS) and electrokinetic potential measurements were carried out. The obtained results show, through a very apparent decrement in anodic current responses, the influence of different organic acid precipitants on nanoink destabilization.
\end{abstract}

Keywords: conductive ink chemistry, cyclic voltammetry, electrochemical impedance spectroscopy, silver nanoparticle, weak organic acids.

\section{INTRODUCTION}

$\mathbf{N}$ OWADAYS, synthesis of metal nanoparticles is generating a lot of research work because of their utilization in fields of catalysis, ${ }^{[1-3]}$ biomedicine, ${ }^{[4,5]}$ optics $^{[6]}$ and electronics. ${ }^{[7,8]}$ Most of the applications are due to the fact that metal particles in the nanometre size have drastically different properties compared to the same bulk material. For this reason, a need for developing novel synthetic routes is required. General mechanisms for producing nanoparticles can be roughly divided into two main approaches. ${ }^{[9]}$ The first method can be described as „bottom up", which relies on building nanoparticles from molecules or atoms. The second one, called „top down“ means to break bulk material down to the nanoscale. Both approaches play very significant roles in nanoscience and nanotechnology. Among all synthetic routes, the one most represented in the literature is the chemical reduction method. Besides the metal precursor, synthesis in a batch requires the presence of a reducing and grain-stabilizing molecule. This procedure is easy to handle, cost-efficient and does not require state of the art equipment. Furthermore, synthesis can be carried out in various ways, by changing reaction parameters and equipment, or using aqueous and/or organic solvents. ${ }^{[10,11]}$

Compared to other noble metals, nanosilver exhibits superior optical and electric properties which makes it a unique material for biosensor applications, ${ }^{[12]}$ antimicrobial coatings ${ }^{[13]}$ and conductive suspensions for inkjet technology. ${ }^{[14]}$ In case of silver particles, nanocrystals are usually produced by reduction of a soluble silver(I) salt. ${ }^{[15-17]}$ Wet chemistry method is the typical synthetic preparation route of AgNPs for conductive ink formulations. The relatively large electropositive reduction potential for silver determines the usage of reductant required for a successful 
chemical process. Among reducing agents, commonly used inorganic compounds are hydrazine, ${ }^{[18]}$ sodium borohydride ${ }^{[19]}$ and trisodium citrate. ${ }^{[20]}$ Organic reductants are often multifunctional, because they can behave as carrier media, [21] surfactants, ${ }^{[22,23]}$ or both. ${ }^{[24]}$ For stability of colloidal silver solutions, produced by this approach, presence of a capping (stabilizing) agent is essential. Stabilizers can be various ionic species adsorbed on the nanocrystal surface, or polymer molecules which cover the nanocrystal surface to prevent particles to collapse. The choice of grain-covering molecules can be very wide and predestine the nanograin application. ${ }^{[25,26]}$

In this paper, we report the synthesis of nanosilver intended for formulation of conductive ink.[27] Ink chemistry is the most important, but also the most demanding step in terms of inkjet technology. For an excellent conductive ink, few requirements should be fulfilled. Firstly, ink should be composed of stabilized AgNPs with narrow size distribution, to enable continuous drop ejecting and to avoid nozzle clogging. Secondly, in order to achieve high conductivity of the printed pattern, concentrated silver nanosol is required. ${ }^{[28]}$ Hence, we based our two-step AgNPs synthetic process on silver nitrate reduction by hydrazine and with poly(acrylic acid) as a stabilizing polymer. The powdered product, the main ingredient for conductive ink formulation, was collected after precipitation by different weak organic acids. According to the literature, sedimentation of nanosilver has been mainly performed with large quantities of acetone ${ }^{[29]}$ or ethanol. ${ }^{[30]}$

The common nanoparticle characterization techniques reported in the literature rely on ultraviolet-visible spectrophotometry, electron microscopy and zeta-potential measurements; consequently, our electrochemical approach is novel. In addition, during two-stage nanoparticle syntheses, weak organic acids have never been described as a sedimentation agent. Therefore, in terms of organic acid impact on surface-bound stabilizer, no study has been done before.

\section{EXPERIMENTAL SECTION}

\section{Materials and Chemicals}

Silver nitrate $\left(\mathrm{AgNO}_{3}\right)$, as a metal precursor in all synthesis experiments done, was obtained from VWR Chemicals (Belgium). Poly(acrylic acid) (PAA, $M_{\mathrm{w}}=1800$ ), ethylene glycol (EG; anhydrous, $w=99.8 \%$ ), hydrazine hydrate solution ( $w=50-60 \%$ ) and 2-amino-2-methyl-1-propanol (2-AMP) were purchased from Sigma-Aldrich (USA). Weak organic acids were gained from: oxalic acid - Kemika (Croatia), citric acid - Gram Mol (Croatia), ascorbic acid Kemika (Croatia), glacial acetic acid-BDH Prolabo (UK) and succinic acid - Kemika (Croatia). Britton-Robinson (BR) buffer solutions were composed of boric acid (0.04 M), phosphoric acid $(0.04 \mathrm{M})$ and acetic acid $(0.04 \mathrm{M})$, and the $\mathrm{pH}$ adjusted using $5 \mathrm{M} \mathrm{NaOH}$ solution. All chemicals needed for BR buffer solutions were obtained from Kemika (Croatia). All applied chemicals were of analytical grade and were used as received. Deionised water, purified using a Millipore-MilliQ system, was used in all of the experiments.

\section{Instrumentation}

Cyclic voltammograms (CVs) were obtained using an EG\&G PAR Model 264A Polarographic Analyser (USA) and EDAQ e-coder (Australia) connected to a PC for data control and data acquisition. A three-electrode electrochemical cell, consisted of a glassy carbon disc electrode (GC, $d=3 \mathrm{~mm}$ ) as a working electrode, $\mathrm{Ag} \mid \mathrm{AgCl} \| 3 \mathrm{M} \mathrm{KCl}$ as a reference electrode and a Pt wire as supporting electrode. All electrochemical experiments were carried out in a $10 \mathrm{~mL}$ electrochemical cell at room temperature, at a scan rate of $50 \mathrm{mV} / \mathrm{s}$, with starting potential at $-0.3 \mathrm{~V}$. Sample homogenization was done by a classic bar stirrer for 15 seconds before measurements.

Electrochemical impedance spectroscopy (EIS) study was carried out on a EG\&G PAR Model 263A Polarographic Analyser (USA), connected to a frequency response detector, applying single-sine technique. Same electrochemical cell, needed for CV measurements, was used also for EIS analysis.

The particle size distribution analysis (Dynamic light scattering method, DLS), as well as the electrokinetic potential measurements of the coated silver NPs, were performed using a Zeta Plus (Brookhaven Instruments Corporation, USA) instrument.

Homogenization of the prepared conductive inks was performed using Bandelin electronic Sonorex high power ultrasound bath (Germany) and Stuart SA8 vortex mixer (UK).

Gilson Miniplus Evolution peristaltic pump fitted with a MF4 pump head (France) was used for accurate addition of the hydrazine hydrate solution to the reaction mixture.

\section{Synthesis of Silver NP in Aqueous Solution}

In this paper, wet chemistry method was chosen to produce silver nanoparticles as a base for formulating the conductive ink. The same synthesis procedure was studied in detail and optimized in our previous work. ${ }^{[31]}$ In contrast to the reported recipe, PAA-protected AgNPs were agglomerated by adding different weak organic acids into a reaction mixture divided in five beakers: oxalic acid (0.01 $\mathrm{M})$, citric acid (0.1 M), ascorbic acid (1 M), acetic acid (3 M) and succinic acid $(1 \mathrm{M})$, respectively. All acids were 
prepared in different molar concentrations; nonetheless, aqueous solutions were of the same $\mathrm{pH}$ value and had the same influence on particle aggregation. Precipitated nanosilver was washed with water and acetone. Wet sediment was dried at $60{ }^{\circ} \mathrm{C}$ after which the silver nanopowder was stored in a glass bottle at room temperature.

\section{Preparation of Stable Conductive Ink Suspension}

The conductive inks, intended for printing technology applications, are usually made of polymer-coating stabilized nanopowder dispersed in a corresponding solvent. Silver nanoparticle suspension was made by mixing the dried PAA-AgNPs into an EG solution (mass fraction, $w=20 \%$ ). Also, small amount of 2-AMP was added for adjusting the suspension's $\mathrm{pH}$ value up to 10.50 . To assure the maximum dispersion of nanoparticles with slight sedimentation, suspensions were vortexed and kept in an ultrasonic bath until homogenization was achieved. Five different nano-ink formulations were prepared, regarding to 5 distinctive organic acids used as a sedimentation agent. In addition, a $6^{\text {th }}$ specimen needed for comprehensive analysis was the sample of AgNPs before precipitation. Mass fraction of stabilized nanometal in all formulations was $3.46 \%$.

\section{RESULTS AND DISCUSSION}

\section{Electrochemical Characterization of Polymer-protected Silver NPs Suspension}

Prior to synthesis start, reaction parameters need to be optimised in order to improve monitoring of the redox process between the silver precursor and hydrazine molecules. Electroanalytical response of the glassy carbon disc electrode in silver and hydrazine solutions was described by applying cyclic voltammetry as a measuring technique. ${ }^{[31]}$ As the oxidation process of metallic silver on the glassy carbon electrode starts at a lower potential than one needed for the hydrazine molecule to oxidize, this electrode can be used as a sensor for monitoring this synthetic process.

Figure 1 depicts the cyclic voltammograms obtained with a GC electrode in a primary buffer solution $(\mathrm{pH}=$ 10.00) and with addition of the reaction mixture sample. The applied potential scan rate was $50 \mathrm{mV} / \mathrm{s}$. Near the equivalence point, our specimen consists of two different silver forms - polymer-stabilized nanometal one, and a positively charged ion. In alkaline media, carboxylic group, as the functional group of poly(acrylic acid), is deprotonated, generating negative charge on the nanometal

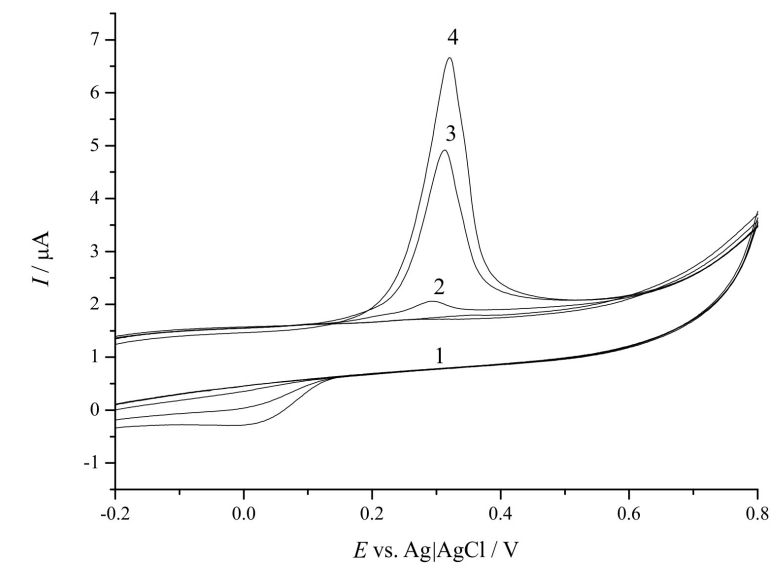

Figure 1. Cyclic voltammograms (CVs) obtained in the BR buffer solution ( $\mathrm{pH}=10.00)$ after the addition of AgNPs (50 $\mu \mathrm{L}$ ) into a $10 \mathrm{~mL}$ cell. Curves $4-2$ represents excess of $\mathrm{Ag}^{+}$in the reaction mixture during reduction; 1 - BR solution. Recorded using a GC working electrode, Pt auxiliary electrode and $\mathrm{Ag}|\mathrm{AgCl}| \mid 3 \mathrm{M} \mathrm{KCl}$ reference electrode. Scan rate was $50 \mathrm{mV} / \mathrm{s}$.

surface. As the working electrode is negatively charged, only detection of cationic species is possible. Hence, the resulting voltammograms are product of unreacted redox couple silver/silver ion. CV denoted with number $\mathbf{4}$ has the highest anodic current response, therefore depicts large excess of silver cation presence in solution. CV curves $\mathbf{3}$ and 2 follow the peak current reduction as a result of hydrazine addition to the reaction mixture. Finally, when the reaction suspension gives a current response presented as voltammogram 1 , which is the same as the pure primary electrolyte solution, we can conclude that the equivalence point in the redox process is reached.

Although hydrazine is an explosive and hazardous molecule, ${ }^{[32]}$ it is also a low-cost, highly available chemical and a fast reduction agent, which renders small nanoparticles with unique optoelectronical and physicochemical properties. In recent years there have been a lot of published scientific articles regarding green nanosilver preparation, ${ }^{[33-35]}$ but our synthetic route is a strictly controlled process which ensures that all of the reducing agent transfers into elementary nitrogen. Hence, there is no indication of releasing the toxic molecule into the environment.

Poly(acrylic acid), adsorbed on the nanometal surface, acts as a grain-stabilizing agent due to the existence of carboxylic groups. ${ }^{[30]}$ This functional group, depending on the $\mathrm{pH}$ value of the medium, can be protonated or deprotonated, describing this polymer behaviour as a weak acid. ${ }^{[36,37]}$ Negative surface charge generates Columbic repulsion forces between nanograins, thus, efficiency of electrostatic stabilization of the prepared 

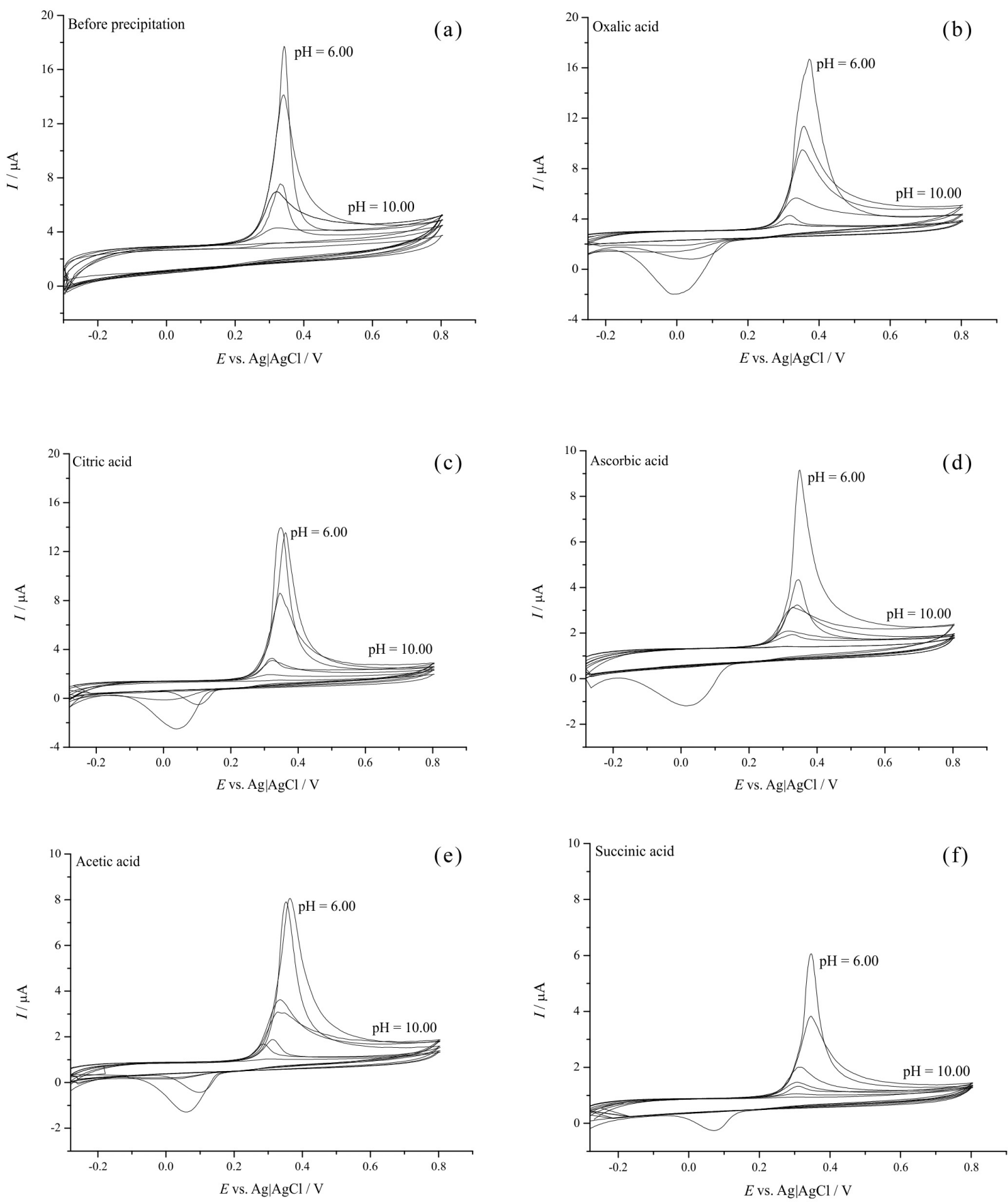

Figure 2. Hydrodinamic CVs of PAA-stabilized AgNPs in: non-precipitated sample (a); conductive inks prepared after nanograin precipitation with oxalic, citric, ascorbic, acetic and succinic acid, (b-f) respectively. Voltammograms were obtained by dissolving $75 \mu \mathrm{L}$ of nanosilver specimen into a $10 \mathrm{~mL}$ of BR buffer solutions at various $\mathrm{pH}$ values. The $\mathrm{pH}$ range changes from 10.00 (low current responses) to 6.00 (the highest current peaks), with $0.5 \mathrm{pH}$ unit decrement.

suspension depends on the acid dissociation rate. Stability of the prepared nano-ink, as well as the AgNPs before precipitation using weak organic acids, were tested in Britton-Robinson buffer solutions, from alkaline $(\mathrm{pH}=$ 10.50) to acidic ( $\mathrm{pH}=4.50)$ media. Obtained hydrodynamic
CVs, recorded in the $\mathrm{pH}$ range between $\mathrm{pH}=10.00$ and $\mathrm{pH}=6.00$ for all formulations done, are shown in Figure 2 a-f. Starting the measurement set at a negative potential, and in high alkaline media, the repulsion force between the electrode and nanograins results in minor current 

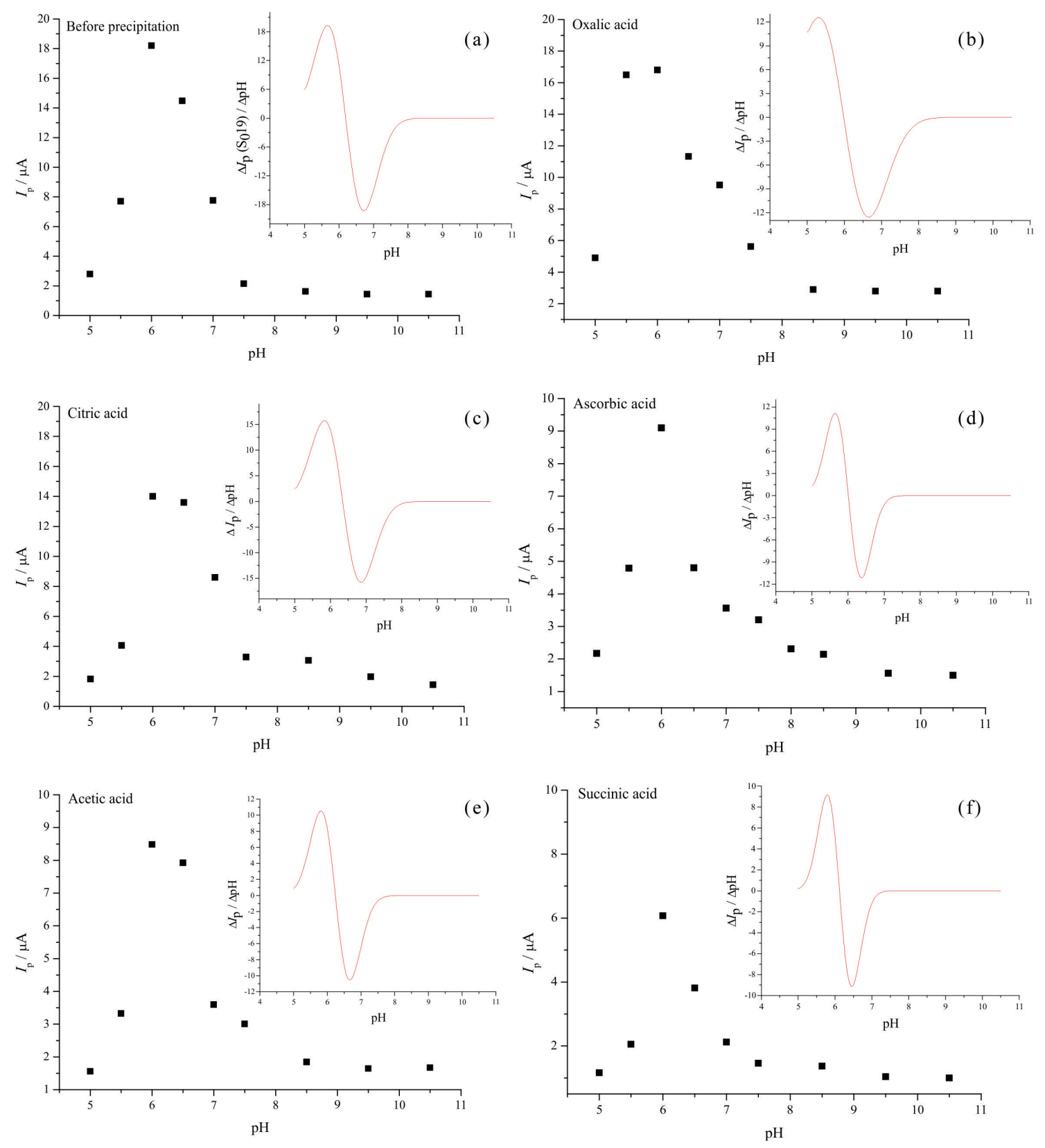

Figure 3. The dependence of the highest anodic current responses, for data shown in Figure 2, on pH of the medium. Inlay: first derivative of the data for each measurement done.

responses. As the polyacid dissociation rate decreases while lowering the $\mathrm{pH}$ value of the medium, higher anodic responses can be noticed. The curve through for all samples is observed at the same $\mathrm{pH}$ value of 6.00 .

For better understanding of this process, highest anodic currents were plotted versus $\mathrm{pH}$ value of the medium for each nanosilver specimen (Figure $3 \mathrm{a}-\mathrm{f}$ ). Starting from the alkaline medium, empirical data gradually rises up to the maximum current value noticeable at $\mathrm{pH}=$ 6.00, after which they exhibit a steep fall. There are three significant data regions in these graphical plots to consider: a) stabilization part, as a consequence of low current responses obtained in alkaline media; b) destabilization of the suspension due to the increment in anodic currents from neutral to slightly acidic media; and c) total disintegration of homogenous suspension at the $\mathrm{pH}$ below 6.00. As approximately the same values of current responses were achieved in both stabilization and fragmentation zone, we can conclude that the current intensity strongly depends, beside the nanosilver 
concentration and dissociation rate of the adsorbed stabilizer, on the $\mathrm{pH}$ value of the media. These presented curves can be used to determine surface bound PAA dissociation constant. All experimental data were extrapolated applying Gauss function in Origin software, and the first derivative curves (Figure $3 \mathrm{a}-\mathrm{f}$; inlay) were done. The minimum of the first derivative function indicates the $\mathrm{p} K_{\mathrm{a}}$ value of the polyelectrolyte. Calculated dissociation constant, gained from CV measurements, for the reaction mixture sample was 6.70 , and for specimens precipitated with oxalic, citric, ascorbic, acetic and succinic acid was 6.60,6.80,6.70,6.40, and 6.50, respectively. These values display a difference from data obtained for free acrylic acid solution $\left(p K_{a}=4.50\right),{ }^{[38]}$ but are in agreement with $\mathrm{p} K_{\mathrm{a}}$ value of 6.50 for homopolymer consisted of 1800 monomer units. ${ }^{[39]}$

Stability of poly(acrylic acid) coated AgNPs, describing its dissociation constant rate, can be determined also by plotting zeta-potential vs. $\mathrm{pH}$ dependence. ${ }^{[30]}$ However, electrokinetic potential does not itemize which part of chemical composition contributes to the suspension's stability. From cyclic voltammetry analysis, we can confirm that prepared metal NPs were successfully facilitated with the polyacidic stabilizator used. Also, knowing from the literature that citric and ascorbic acids are described as reducing and stabilizing agents, ${ }^{[11]}$ we can claim that no binding of organic acid to form amphiphilic nanoparticles occurred. Otherwise, the gained $I_{\mathrm{p}} / \mathrm{pH}$ curve would have two inflection points and would have been the same as a titration curve of a diprotic acid.

Because of the fact that maximum current responses for all homogenous suspensions are noticed at $\mathrm{pH}=6.00$, we decided to use this BR buffer solution as a base electrolyte solution for further characterization of the system. Figure 4 demonstrates hydrodynamic CVs of the PAA-stabilized AgNPs obtained in this BR buffer solution. Curve 1, which designates non-precipitated nanosilver solution, has the highest current peak. It can be seen that, by adding a precipitation agent (curves 2-6), the anodic peak is slightly shifted toward lower potentials. Furthermore, implementation of weak organic acids expresses evident peak current diminution (Figure 5). Therefore, precipitation of nanosilver by different organic acids was efficient and had visible impact on the suspension stability. We can conclude that with introduction of a precipitation agent into a reaction mixture some amount of adsorbed polyacid is removed from the nanograin surface, causing slight destabilization of AgNP ink formulation.

Alongside to CV measurements, impedance spectroscopy technique is also a powerful tool in the study of electrochemical systems. For detailed understanding of the conductive ink properties, EIS study was done. Experiments were conducted on the same specimens used in CV

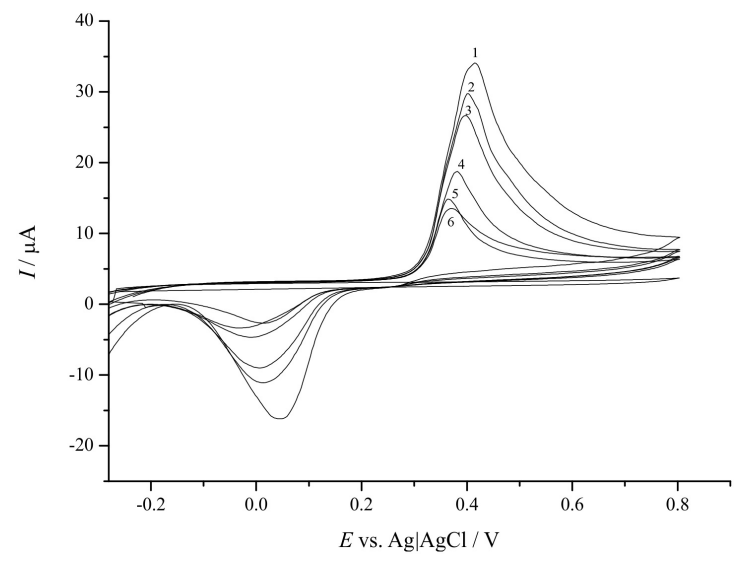

Figure 4. Hydrodinamic CVs of the prepared nano-ink, $w($ PAA-AgNP $)=3.46 \%$, obtained by adding $150 \mu \mathrm{L}$ of nanosilver in a $10 \mathrm{~mL} B R$ buffer solution $\mathrm{pH}=6.00$. Curve denoted 1 depicts specimen at the end of the nanosilver synthesis; CVs represent AgNPs precipitated with weak organic acids: oxalic (2), citric (3), ascorbic (4), acetic (5), succinic (6). Measurement set up was the same as in Figure 1.

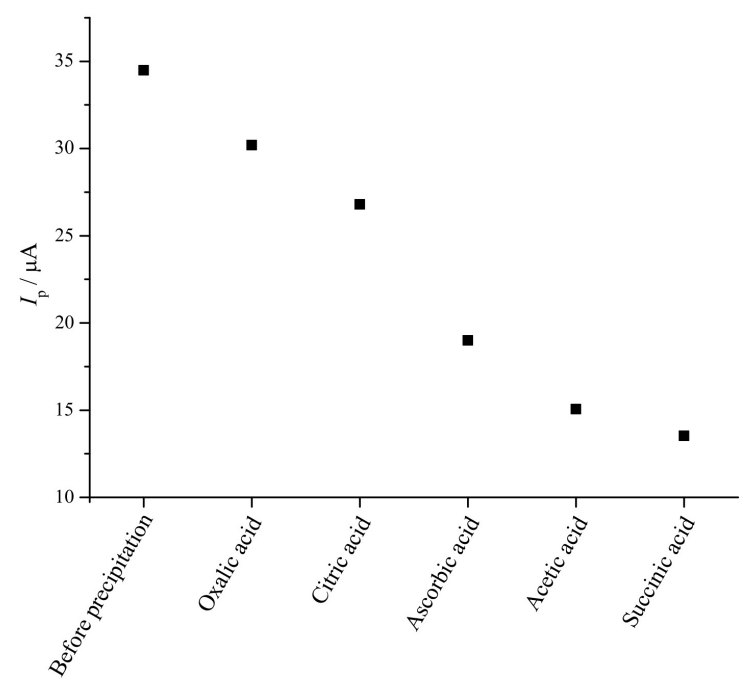

Figure 5. Highest anodic currents, for data shown in Figure 3, express evident peak current diminution, as a repercussion of organic acid addition to the reaction mixture.

analysis. Also, measurement setup, composed of threeelectrode electrochemical cell, was equivalent. Figure 6 presents impedance spectra of non-precipitated nanosilver solution and formulations of redispersed AgNPs.

The highest real impedance parameters, determined on the top of semicircle in Nyquist diagram, for all measurements done were presented in Figure 7. It can be observed that the impedance resistance values are reversely proportional to the maximum current response gained with CV technique. 


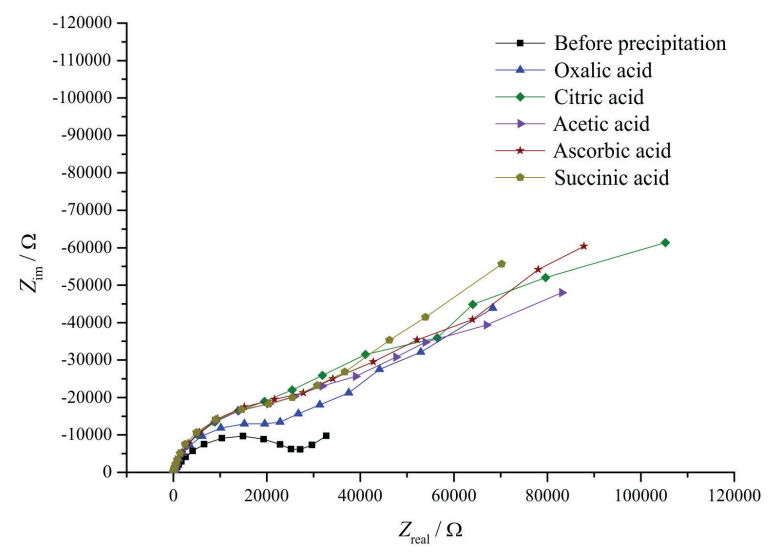

Figure 6. Nyquist graphical plot of stabilized nanosilver suspension, $w($ PAA-AgNP) $=3.46 \% .150 \mu \mathrm{L}$ of conductive ink formulation was mixed with $10 \mathrm{~mL}$ BR buffer solution of $\mathrm{pH}$ value 6.00. Impedance measurements were conducted on the same samples used in CV analysis. All experiments were measured at $D C$ potential $(E=310 \mathrm{mV})$ and $A C$ sine wave with $10 \mathrm{mV}$ of amplitude was applied. Impedance spectra were collected in the frequency range between $1 \mathrm{MHz}$ to 1 $\mathrm{mHz}$.

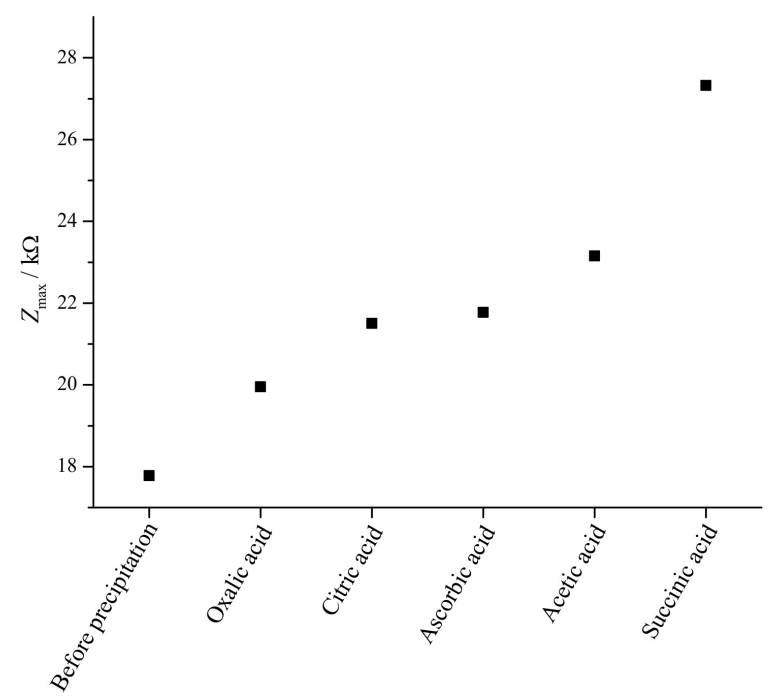

Figure 7. Graphical plot of the highest impedance parameters read from Nyquist diagram.

\section{DLS and Zeta-Potential Analysis}

In conductive inks intended for inkjet printing, the major requirement which needs to be fulfilled is to have nanoparticles small in size to avoid clogging of printer nozzles. ${ }^{[40]}$ This implies that the nanometal preparation procedure has to be strictly controlled. After redispersion of AgNPs in an appropriate carrier, size distribution of the particles was investigated by dynamic light scattering method (Figure 8). The figure clearly shows that the
Table 1. Average particle diameter and corresponding zetapotential values for non-precipitated nanosilver suspension and formulations obtained after precipitation with weak organic acids.

\begin{tabular}{ccc}
\hline Sample & $d / \mathrm{nm}$ & $\zeta / \mathrm{mV}$ \\
\hline Non-precipitated AgNP & $1.90 \pm 0.03$ & $-52.83 \pm 1.29$ \\
Oxalic acid & $2.00 \pm 0.03$ & $-44.86 \pm 1.77$ \\
Citric acid & $1.80 \pm 0.03$ & $-46.92 \pm 0.83$ \\
Ascorbic acid & $2.70 \pm 0.01$ & $-44.89 \pm 1.39$ \\
Acetic acid & $2.60 \pm 0.02$ & $-40.55 \pm 1.10$ \\
Succinic acid & $1.80 \pm 0.02$ & $-46.13 \pm 1.27$ \\
\hline
\end{tabular}

prepared AgNPs are small in size for all samples, with the average diameter being about $2 \mathrm{~nm}$. Also, narrow size distribution is achieved. The obtained nanoparticles are generally smaller than those found in conductive inks for inkjet printing. ${ }^{[29,30,41]}$

In the field of colloidal dispersion stability, an indispensable tool that should not be avoided is measurement of the electrokinetic potential. ${ }^{[42]}$ Zetapotential value is a number that gives the tendency of particles to agglomerate or to sedimentate, but it says nothing about chemical composition of the solution. For homogenous suspensions consisting of negatively charged particles, it is considered that good cohesion is achieved below potentials of $-25 \mathrm{mV}$. So, the stability of the prepared PAA-AgNPs in the reaction mixture, as well as the ink formulation of redispersed particles, has been confirmed with zeta-potential evaluation. DLS and electrokinetic potential measurements are carried out by the same instrument, so the gathered results are summarized in Table 1.

It is evident that the most stable nanosilver suspension is obtained in the reaction mixture at the end of the synthesis. By adding the precipitation agent, the measured zeta-potential is to some extent lower and can be attributed to slight particle enlargement but, for all formulations, stays in the high stability constituency.

\section{CONCLUSIONS}

We have developed a conductive silver nanoparticle basedink by applying reduction of aqueous nitrate solution with hydrazine monohydrate. Poly(acrylic acid), used as a protection polymer, has influence on grain size and stability. Cyclic voltammetry can be successfully applied in all stages of process regulation. Firstly - for preliminary examination of the reaction parameters in order to provide an easy, fast and economic synthetic procedure. Secondly 

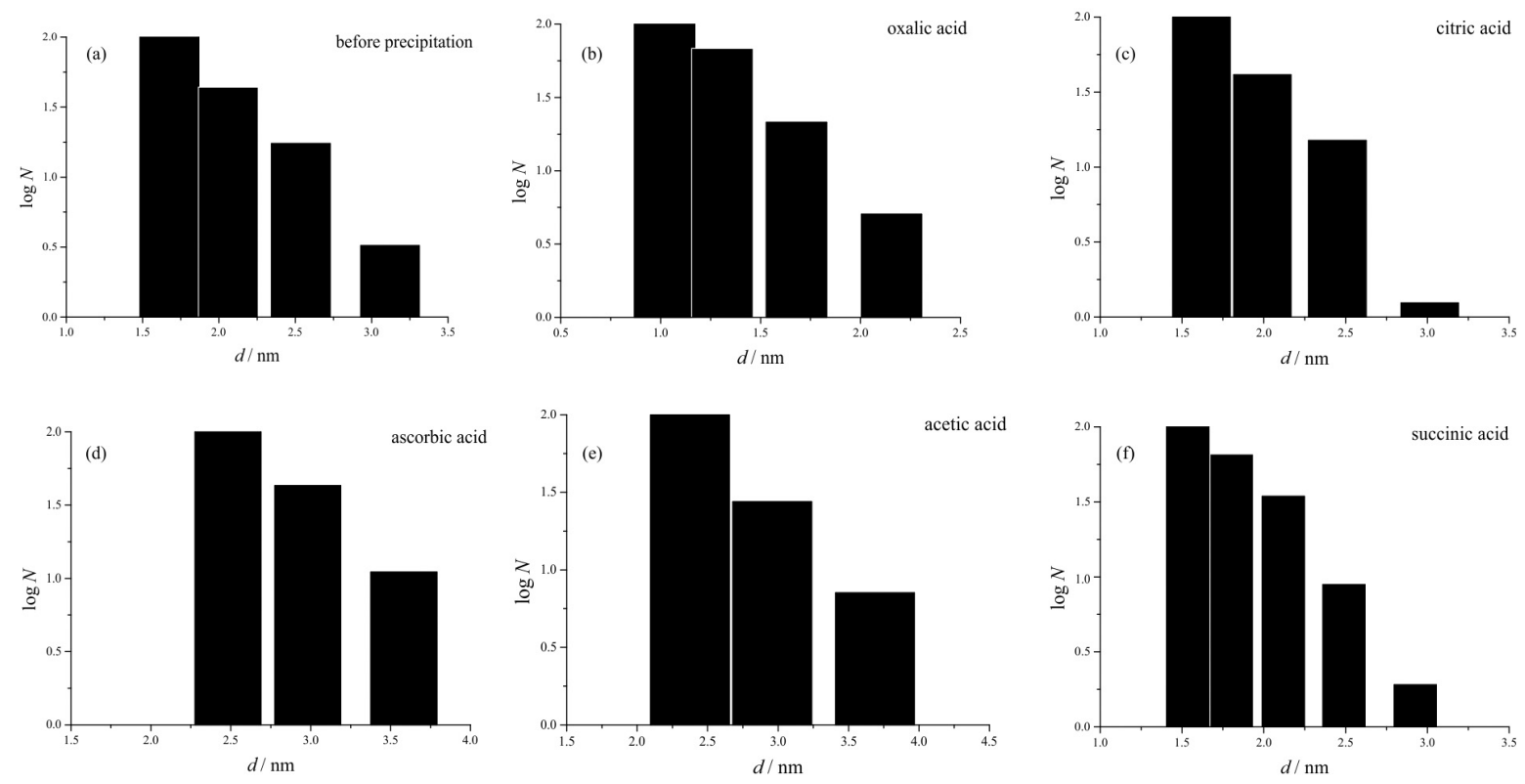

Figure 8. Particle size distribution of PAA-stabilized AgNPs: before (a) and after precipitation using oxalic (b); citric (c); ascorbic (d); acetic (e); succinic acid (f). Sample dilution for all specimens was $\varphi=1: 30000$.

- for precise control of the reactants stoichiometric ratio, in purpose of obtaining high nanosilver yield and, at the same time, avoiding excess of harmful reactants in the ecosystem. Thirdly - for characterizing the ink stability in a wide $\mathrm{pH}$ range, and finally - for determining the existence of surface bound-polyacid. For detailed study of the nanosilver dispersion, electrochemical impedance spectroscopy was used as another characterization method. This work provides a new characterization insight of a nanometal-based ink chemistry field. By comparing the dissociation constant values of non-precipitated sample $\left(\mathrm{p} K_{\mathrm{a}}=6.70\right)$ and nanoink formulations prepared after precipitation with weak organic acids $\left(\mathrm{p} K_{\mathrm{a}}=6.60 \pm 0.20\right)$, we can assume that no binding of precipitants on the nanograin occurred. Noticeable impact can be seen in detected anodic peak currents sequence, starting from oxalic acid (highest peak obtained) to succinic acid with lowest current trough. Anodic current decrement suggests slight nanoparticle aggregation and negligible loss of adsorbed stabilizer. This behaviour is also confirmed in minor average grain diameter enlargement, and in lower measured electrokinetic potential values.

Acknowledgment. The authors are grateful to the University of Zagreb (Grant No. 118020) for financial support. Special thanks to Prof. Tajana Preočanin from the Faculty of Science, University of Zagreb, for the use of the Zeta Plus instrument.

\section{REFERENCES}

[1] S. B. Kalidindi, B. R. Jagirdar, ChemSusChem 2012, 5, 65.

[2] S. Jana, Dalton Trans. 2015, 44, 18692.

[3] P. Viswanathan, R. Ramaraj, Polym. Int. 2017, 66, 342.

[4] T. Cohen-Karni, R. Langer, D. S. Kohane, ACS Nano 2012, 6, 6541.

[5] G. Maduraiveeran, M. Sasidharan, V. Ganesan, Biosens. Bioelectron. 2018, 103, 113.

[6] I. Fratoddi, Nanomaterials 2018, 8, 25.

[7] W. Lu, C. M. Lieber, Nat. Mater. 2007, 6, 841.

[8] S. Wunscher, R. Abbel, J. Perelaer, U. S. Schubert, J. Mater. Chem. C 2014, 2, 10232.

[9] I. Capek, Noble Metal Nanoparticles, Springer, New York, 2017, p. 125.

[10] A. P. Reverberi, N. T. Kuznetsov, V. P. Meshalkin, M. Salerno, B. Fabiano, Theor. Found. Chem. Eng. 2016, $50,59$.

[11] N. L. Pacioni, C. D. Borsarelli, V. Rey, A. V. Veglia, in Silver Nanoparticle Applications: In the Fabrication and Design of Medical and Biosensing Devices, (Eds.: E. I. Alarcon, M. Griffith, K. I. Udekwu) Springer International Publishing, Switzerland, 2015, pp. 13-46.

[12] R. Salahandish, A. Ghaffarinejad, S. M. Naghib, K. Majidzadeh-A, H. Zargartalebi, A. Sanati-Nezhad, Biosens. Bioelectron. 2018, 117, 104. 
[13] L. B. Qi, Z. Y. Liu, N. Wang, Y. X. Hu, Appl. Surf. Sci. 2018, 456, 95.

[14] B. H. Ryu, Y. Choi, H. S. Park, J. H. Byun, K. Kong, J. O. Lee, H. Chang, Colloid Surf. A-Physicochem. Eng. Asp. 2005, 270, 345.

[15] Z. L. Zhang, W. Y. Zhu, J. Alloy. Compd. 2015, 649, 687.

[16] S. Murthy, T. P. Bigioni, Z. L. Wang, J. T. Khoury, R. L. Whetten, Mater. Lett. 1997, 30, 321.

[17] A. Bafana, S. V. Kumar, S. Temizel-Sekeryan, S. A. Dahoumane, L. Haselbach, C. S. Jeffryes, Sci. Total Environ. 2018, 636, 936.

[18] B. Swain, D. Shin, S. Y. Joo, N. K. Ahn, C. G. Lee, J. H. Yoon, Chemosphere 2018, 194, 793.

[19] P. Mendis, R. M. de Silva, K. M. N. de Silva, L. A. Wijenayaka, K. Jayawardana, M. Yan, RSC Adv. 2016, 6, 48792.

[20] Z. S. Pillai, P. V. Kamat, J. Phys. Chem. B 2004, 108, 945.

[21] D. Kim, S. Jeong, J. Moon, Nanotechnology 2006, 17, 4019.

[22] M. Chen, Y. G. Feng, X. Wang, T. C. Li, J. Y. Zhang, D. J. Qian, Langmuir 2007, 23, 5296.

[23] S. Papp, R. Patakfalvi, I. Dekany, Croat. Chem. Acta 2007, 80, 493.

[24] A. Q. Zhang, L. J. Cai, L. Sui, D. J. Qian, M. Chen, Polym. Rev. 2013, 53, 240.

[25] C. C. Li, S. J. Chang, F. J. Su, S. W. Lin, Y. C. Chou, Colloid Surf. A-Physicochem. Eng. Asp. 2013, 419, 209.

[26] S. Miljanić, M. Ratkaj, I. Avdejev, K. Meglić, A. Kenđel, Croat. Chem. Acta 2015, 88, 387.
[27] Z. L. Zhang, X. Y. Zhang, Z. Q. Xin, M. M. Deng, Y. Q. Wen, Y. L. Song, Nanotechnology 2011, 22, 8.

[28] P. Calvert, Chem. Mat. 2001, 13, 3299.

[29] B. Y. Ahn, J. A. Lewis, Mater. Chem. Phys. 2014, 148, 686.

[30] Q. J. Huang, W. F. Shen, Q. S. Xu, R. Q. Tan, W. J. Song, Mater. Chem. Phys. 2014, 147, 550.

[31] S. Milardović, I. Ivanišević, A. Rogina, P. Kassal, Int. J. Electrochem. Sci. 2018, 13, 11136.

[32] K. H. Nguyen, Y. Q. Hao, W. S. Chen, Y. T. Zhang, M. T. Xu, M. H. Yang, Y. N. Liu, Luminescence 2018, 33, 816.

[33] S. B. Jaffri, K. S. Ahmad, Rev. Inorg. Chem. 2018, 38, 127.

[34] E. Roy, S. Patra, S. Saha, D. Kumar, R. Madhuri, P. K. Sharma, Chem. Eng. J. 2017, 321, 195.

[35] S. J. P. Jacob, V. L. S. Prasad, S. Sivasankar, P. Muralidharan, Food Chem. Toxicol. 2017, 109, 951.

[36] A. Sadeghpour, A. Vaccaro, S. Rentsch, M. Borkovec, Polymer 2009, 50, 3950.

[37] T. Swift, L. Swanson, M. Geoghegan, S. Rimmer, Soft Matter 2016, 12, 2542.

[38] S. W. Cranford, M. J. Buehler, Soft Matter 2013, 9, 1076.

[39] M. Wisniewska, T. Urban, E. Grzadka, V. I. Zarko, V. M. Gun'ko, Colloid Polym. Sci. 2014, 292, 699.

[40] M. C. Dang, T. M. D. Dang, E. Fribourg-Blanc, Adv. Nat. Sci.-Nanosci Nanotechnol 2015, 6, 8.

[41] W. F. Shen, X. P. Zhang, Q. J. Huang, Q. S. Xu, W. J. Song, Nanoscale 2014, 6, 1622.

[42] N. Kallay, T. Preočanin, D. Kovačević, J. Lützenkirchen, E. Chibowski, Croat. Chem. Acta 2010, 83, 357. 